\title{
An Accurate Integral Method for Vibration Signal Based on Feature Information Extraction
}

\author{
Yong Zhu, ${ }^{1,2}$ Wanlu Jiang, ${ }^{1,2,3}$ Xiangdong Kong, ${ }^{1,2}$ Zhi Zheng, ${ }^{1}$ and Haosong $\mathrm{Hu}^{2}$ \\ ${ }^{1}$ Hebei Provincial Key Laboratory of Heavy Machinery Fluid Power Transmission and Control, Yanshan University, \\ Qinhuangdao, Hebei 066004, China \\ ${ }^{2}$ Key Laboratory of Advanced Forging \& Stamping Technology and Science, Yanshan University, Ministry of Education of China, \\ Qinhuangdao, Hebei 066004, China \\ ${ }^{3}$ College of Mechanical Engineering, Yanshan University, Qinhuangdao, Hebei 066004, China
}

Correspondence should be addressed to Wanlu Jiang; wljiang@ysu.edu.cn

Received 16 June 2014; Revised 10 September 2014; Accepted 23 September 2014

Academic Editor: Nuno M. Maia

Copyright (C) 2015 Yong Zhu et al. This is an open access article distributed under the Creative Commons Attribution License, which permits unrestricted use, distribution, and reproduction in any medium, provided the original work is properly cited.

\begin{abstract}
After summarizing the advantages and disadvantages of current integral methods, a novel vibration signal integral method based on feature information extraction was proposed. This method took full advantage of the self-adaptive filter characteristic and waveform correction feature of ensemble empirical mode decomposition in dealing with nonlinear and nonstationary signals. This research merged the superiorities of kurtosis, mean square error, energy, and singular value decomposition on signal feature extraction. The values of the four indexes aforementioned were combined into a feature vector. Then, the connotative characteristic components in vibration signal were accurately extracted by Euclidean distance search, and the desired integral signals were precisely reconstructed. With this method, the interference problem of invalid signal such as trend item and noise which plague traditional methods is commendably solved. The great cumulative error from the traditional time-domain integral is effectively overcome. Moreover, the large low-frequency error from the traditional frequency-domain integral is successfully avoided. Comparing with the traditional integral methods, this method is outstanding at removing noise and retaining useful feature information and shows higher accuracy and superiority.
\end{abstract}

\section{Introduction}

The vibration parameters such as acceleration, velocity, and displacement have been commonly utilized in the mechanical fault diagnosis and condition monitoring [1]. At present, the vibration velocity (VV) and vibration displacement (VD) can be measured by laser equipment or extensometer, but it is complicated in the actual testing [2]. By contrast, the vibration acceleration (VA) is widely used due to the desired advantages such as small size, convenient installation, and wide frequency range. Nevertheless, in the vibration system analysis, VV and VD are regularly required $[3,4]$. They are also used to analyze the operating condition and health status of machinery. Thus, the integral of vibration signal is involved in many practical engineering and is worthy of further exploration.
The integral process can be realized via hardware or software. Now, many instruments can implement the conversion by using integral circuit. However, the performance parameters of electronic components possess large discreteness. The precision of results will decline and even produce distortion [5]. To ensure the precision and accuracy of hardware-based integration, the calibration and correction are necessary for the signals with different frequency and amplitude. Meanwhile, the integrator with high performance is quite expensive, and multichannels will greatly increase the cost $[1,3,5]$. Currently, with the development of computer and data processing technology, these deficiencies make software-based integration method more attractive.

At present, there are basically two software integral methods. One is time-domain integral (TDI), and the other is frequency-domain integral (FDI) $[2,3]$. Thong et al. 
theoretically analyzed the determinants of root mean square error under the condition of noise, which pointed out the direction to reduce error $[6,7]$. A baseline correction method was based on least squares fitting and could eliminate the trend item in time domain [8]. Zhou et al. described a waveform correction solution based on least squares fitting, which could well remove the primary trend item [9]. Zhang et al. analyzed the error reasons of the software-based integral and discussed the merit and demerit of the high-pass filtering and piecewise polynomial fitting when they were used to eliminate trend item [10]. Hong et al. proposed a TDI method which converted integral problem into a boundary value problem [11].

The FDI method also attracted wide attention. Han et al. pointed out that the error of FDI mainly came from spectral leakage and low-frequency component generated by noise. To reduce the influence of noise, the frequency components whose amplitude was less than direct current component by $6 \mathrm{~dB}$ or more were suggested to be set to zero $[12,13]$. However, a larger error would exist in the integral initial position. On that basis, a frequency fitting method named vibration attenuation integral was further proposed, and the results had been significantly improved [14]. Ribeiro et al. put forward the FFT-DDI method which reduced the influence of the low-frequency component and was highly effective for the case with high signal-to-noise ratio (SNR) [15]. Duan et al. employed a waveform correction algorithm based on timedomain interpolation and fast Fourier transform (FFT) to eliminate trend item [16]. Wen et al. proposed an approach based on signal reconstruction from the perspective of threshold setting, and its effectiveness was laudably proved [17].

Researches above have made remarkable achievements and a lot of beneficial explorations in both TDI and FDI, which provides research basis and ideas for subsequent study. However, in practical application, as a result of the influences of noise and low-frequency component, the integral accuracy still needs to be further improved [17]. Among all of the aforementioned methods, researches mainly focus on eliminating the low-frequency trend item. However, most parts of rotating machinery often contain complex, nonlinear, and nonstationary characteristics. Such features may range from quasiperiodic to completely irregular behaviors. Specifically as a fault machine, the vibration characteristics are varied. It is worth noting that the most important step for implementing fault diagnosis is to find out an appropriate feature, which can accurately represent the variation of vibration signals as the health condition change. However, it was indicated that traditional FFT-based frequency domain methods are not quite suitable for nonstationary signal analysis since the inherent information of nonstationary signals might not be revealed and extracted [18]. Hence, the analysis of nonstationary signals requires specific techniques [19].

In recent years, many time-frequency analysis techniques have been employed in vibration signal analysis. The shorttime Fourier transform (STFT) [20] utilizes a sliding window and applies a stationary signal spectrum analysis on each signal frame. However, restricted by the Heisenberg uncertainty principle (HUP) [21], the trade-off between time resolution and frequency resolution is inevitable. Besides, the window width is fixed and the STFT cannot be suitable for multiresolution analysis in many application fields. On this basis, the wavelet transform (WT) is proposed with a variable window [22]. Nevertheless, there are some disadvantages in WT, such as the appropriate selections of the base function and certain frequency bands with defect information [23]. Since the WT derives from STFT, it is also restricted by HUP and the variable window is not self-adaptive. As a kind of quadratic transform, the Wigner-Ville distribution [24] can achieve a high accurate estimation for monofrequency signals. However, when the signal is nonlinear or multicomponent, cross terms will arise and result in misinterpretation of the signal [25]. Excitedly, a novel technique named ensemble empirical mode decomposition (EEMD) has been proposed [26], which is developed from the popular empirical mode decomposition (EMD) [27]. Recently, it has been proved that EEMD is an effective analysis method for nonlinear and nonstationary signals $[28,29]$. It can decompose any nonlinear and nonstationary signals to several intrinsic mode functions (IMF) with different vibration modes. Thus, it can availably separate the useful components and the noise components [26]. The EEMD algorithm is essentially a sifting process with two main effects. One is to remove the superimposed waves and the other is to make the waveform more symmetrical. Moreover, the decomposition is self-adaptive and each IMF has integrity and orthogonality, which can be utilized to amend waveform. Meanwhile, EEMD has efficiently solved the modal aliasing problem. Although EEMD possesses many virtues, so far few studies have used it to solve the integral problem for vibration signal.

This paper proposes a novel integral method based on feature information extraction (FIE). This method takes full advantage of the unique virtues of EEMD in dealing with nonlinear and nonstationary signals. Moreover, the method merges the superiorities of kurtosis, mean square error, energy, and singular value decomposition on signal feature extraction. Then, the feature information inherent in vibration signal is accurately extracted, and the desired integral signals are precisely reconstructed. With this method, the interference problem of invalid signal such as trend item and noise which plague traditional integral methods is commendably solved. The great cumulative error from the traditional TDI is effectively overcome. Moreover, the large low-frequency error from the traditional FDI is successfully avoided. Comparing with the traditional methods, this method is splendid at removing noise and retaining useful feature information and shows higher accuracy and superiority. The feasibility and effectiveness of the proposed method are verified by the simulation and measured signals.

\section{Basic Principle of Signal Integral}

2.1. Time-Domain Integral Principle. Define $a(t)$ as the acceleration signal. Then, the velocity signal after integration can be expressed as follows:

$$
v(t)=\int_{0}^{t} a(t) d t=\bar{v}(t)+v_{0} .
$$


The displacement signal after integration can be given by

$$
y(t)=\int_{0}^{t} v(t) d t=\bar{y}(t)+y_{0}
$$

where $\bar{v}(t)$ is dynamic velocity component after integration, $\bar{y}(t)$ is dynamic displacement component after integration, $v_{0}$ is static velocity component after integration, and $y_{0}$ is static displacement component after integration, $t$ is time, $d t$ is differential about $t$.

In numerical calculation, the time-domain continuous integral aforementioned is usually replaced with Simpson integral to perform approximate calculation [30]. The velocity calculation formula is

$$
v(k)=v(k-1)+\frac{[a(k-1)+4 a(k)+a(k+1)] T_{s}}{6} .
$$

The displacement calculation formula is

$$
y(k)=y(k-1)+\frac{[v(k-1)+4 v(k)+v(k+1)] T_{s}}{6},
$$

where $k$ is signal sampling points and $T_{s}$ is sampling time.

The vibration signal commonly possesses complex structure and contains multiple components. Before integration, the direct current component of signal must be eliminated to reduce the influence of trend item on result. However, it is impossible to synchronously sample all components with full period in actual test, which leads to an inevitable deviation in subsequently eliminating direct current operation and further the trend item cannot be completely removed [17]. Moreover, the constant terms and linear terms will be generated after integration. Specifically after the second integration, the amplitude of VD will result in large deviation. The cumulative errors from integrals have a considerable effect in the calculation accuracy. Furthermore, the complex noise components mixed in VA signal also cause the complex nonlinear items existing in VV and VD signals. Therefore, the VV and VD directly obtained by integration from VA will originate large deviation, even complete distortion. At present, the primary means to amend TDI is firstly to process the original signal by means of zero-mean processing, which can eliminate the direct current component in the signal. Then, TDI is performed to the preprocessed signal. Finally, the trend item produced in the integral is eliminated by means of polynomial fitting.

2.2. Frequency-Domain Integral Principle. The principle of FDI is firstly to convert the time-domain signal into frequency domain via FFT. Then, the integral operation in time domain is changed into the algebraic operation of spectrum in frequency domain. Moreover, the integral results can be obtained by inverse fast Fourier transform (IFFT).
Consider that $a(n), v(n)$, and $y(n)$ are the discrete forms of the acceleration, velocity, and displacement, respectively. They can be described as follows:

$$
\begin{gathered}
a(n)=\sum_{k=0}^{N-1} A(k) e^{j 2 \pi k n / N}, \\
v(n)=\sum_{k=0}^{N-1} \frac{1}{j \omega_{k}} H(k) A(k) e^{j 2 \pi k n / N}, \\
y(n)=\sum_{k=0}^{N-1} \frac{1}{\left(j \omega_{k}\right)^{2}} H(k) A(k) e^{j 2 \pi k n / N} .
\end{gathered}
$$

Among them,

$$
\begin{gathered}
\omega_{k}=2 \pi k \Delta f, \\
H(k)= \begin{cases}1, & f_{d} \leq k \Delta f \leq f_{u}, \\
0, & \text { others. }\end{cases}
\end{gathered}
$$

Then, the relationships of algebraic operations among them in frequency domain can be determined as follows:

$$
\begin{gathered}
V(k)=\frac{A(k)}{j \omega_{k}}, \\
Y(k)=-\frac{A(k)}{\omega_{k}^{2}},
\end{gathered}
$$

where $A(k), V(k)$, and $Y(k)$ are Fourier transforms of the acceleration, velocity, and displacement, respectively; $\Delta f$ is the frequency resolution; $j$ is the imaginary unit; $H(k)$ is the frequency characteristic of the band-pass filter; $f_{d}$ and $f_{u}$ are the lower and the upper cut-off frequency of the band-pass filter, respectively; $N$ is the number of the sampling points.

According to (7), FDI directly utilizes the algebraic operation relationship of spectrum in frequency domain as the principle. The cumulative error amplification effect can be efficiently avoided. But (7) also indicate that the amplitude of spectrum increases gradually when $\omega_{k}<1 \mathrm{rad} / \mathrm{s}$. Specifically the displacement is from the frequency-domain second integration, and the amplitude of spectrum is related to $1 / \omega_{k}^{2}$. When low frequency is close to zero, it will produce a large low-frequency oscillation and peak error, which exerts low-frequency sensitivity. However, the accuracy in the low band of acceleration sensor is unsatisfactory [3, 30]. As a result, the low band is an important error source of FDI. As a common approach, the components below a certain frequency in spectrum are directly set to zero to get rid of the trend item.

\section{Feature Extraction}

For faulty rotating machinery, the effective components of signals mainly consist of rotational frequency and fault characteristic frequency [17]. In the signal spectrum, the frequency characteristic and the amplitude where spectrum peaks appear are commonly used to estimate the fault type 
and severity [31,32]. This means that, in the vibration signal analysis of faulty rotating machinery, we can focus on a limited number of signal components, that is, rotational frequency, fault characteristic frequency, and its frequency multiplication which includes superharmonic and subharmonic frequency components. Furthermore, other components can be seen as noise to be eliminated.

Kurtosis is a numerical statistic that reflects distribution features of vibration signal. It is usually utilized to quantitatively describe the impact level of vibration signal due to its sensitivity to impact signal [33]. As for discrete signal, its expression can be expressed by

$$
K=\frac{1}{N} \sum_{i=1}^{N}\left(\frac{x_{i}-\mu}{\sigma}\right)^{4},
$$

where $x_{i}$ is sampling signal, $\mu$ is the mean of signal, and $\sigma$ is standard deviation.

Mean square error (MSE) is usually utilized to quantitatively describe calculation or measurement accuracy [34]. As for discrete signal, its expression can be described as

$$
\text { MSE }=\frac{1}{N} \sum_{i=1}^{N}\left(x_{i}-\mu\right)^{2} .
$$

Energy can reflect the strength degree of the whole data set and the energy difference of different waveforms [35]. Hence, the energy value can be used to quantificationally measure the overall effect of different movement types. As for discrete signal, it can be expressed as

$$
E=\sum_{i=1}^{N} x_{i}^{2} .
$$

Singular value reflects the energy distribution of useful signal and noise mixed in the signal. After the signal is processed by singular value decomposition (SVD), a set of singular values will be obtained. Among them, the larger mainly reflects the useful signal, and the smaller primarily reflects the noise. SVD can realize signal denoising. Moreover, the denoising results possess the advantages of zero phase shift, low waveform distortion, and high signal-tonoise ratio [36]. Meanwhile, SVD also does well in feature information separation and weak signal extraction. It can effectively extract the feature information from the signal mixed with noise and can accurately extract weak amplitudemodulation features implied in the vibration signal [37].

In order to effectively extract the useful features of vibration signal, this paper takes full use of the superiorities of kurtosis value, MSE value, energy value, and the largest singular value on the feature extraction and combines the four indexes into a feature vector $\mathbf{T}=\left[\begin{array}{llll}a & b & c & d\end{array}\right]$.

\section{Integral Principle of FIE and Error Evaluation}

4.1. Integral Principle Based on FIE. Based on the aforementioned analysis, this paper proposes a novel integral method based on FIE. The dominant idea of the method is mainly to consider the effective vibration components that reflect signal features during integral process. The feature information of fault characteristics frequency and its superharmonic or subharmonic frequency components are extracted by search and correction. Then, the desired and accurate integral signals are reconstructed with the feature information.

The flow chart of the proposed FIE-based integral method is shown in Figure 1.

The detailed steps are as follows.

(1) Zero-Mean Processing for the First Time. Before integration, the original acceleration signal is processed by means of zero-mean processing, which can eliminate the direct current component mixed in the signal. The aim of this step is to eliminate the interference of trend item on the integral result.

(2) Frequency-Domain Integral for the First Time. In the FDI, (7) indicate that the noise signals are amplified after the integration operation when $\omega_{k}<1 \mathrm{rad} / \mathrm{s}$; but they are suppressed when $\omega_{k} \gg 1 \mathrm{rad} / \mathrm{s}$. In other words, the noise signal whose frequency $f=\omega_{k} / 2 \pi$ is lower than $1 / 2 \pi \approx$ $0.159 \mathrm{~Hz}$ is amplified. However, the vibration signal in the same frequency is not usually useful in engineering practice [3]. Therefore, in order to prevent the noise signal from being amplified in integral process, these components should be set to zero. Moreover, in the actual vibration test, the measure ranges of different sensors are varied. A sensor cannot accurately measure the signal whose frequency is below the lower limit frequency of the sensor. Hence, the signal of this frequency range is mainly noise. Thus, the lower limit test frequency of the sensor should be considered as a crucial part in the integral process. Meanwhile, the frequency components below it should be set to zero.

Based on the discussion above, firstly, set a reasonable cut-off frequency according to the measure sensor and actual test situation. Secondly, the FDI with low-frequency filtering correction is employed to integrate the pretreated acceleration signal. Then, the velocity time-domain signal can be obtained.

(3) Zero-Mean Processing for the Second Time. The velocity time-domain signal is processed by means of zero-mean processing, which can get rid of the direct current component mixed in the velocity signal. The aim of this step is to eliminate the influence of trend item on the subsequent processing results.

(4) EEMD for the First Time. This step takes full advantage of EEMD in dealing with nonlinear and nonstationary signal and decomposes the velocity signal obtained by step (3) to several IMF components which reflect different vibration modes.

In the implementation process, the amplitude of the added white noise and the ensemble number are two critical parameters that need to be determined. These parameters directly affect the decomposition performance of EEMD. Based on the experimental observations, Wu and Huang [26] concluded that increasing noise amplitude and ensemble 


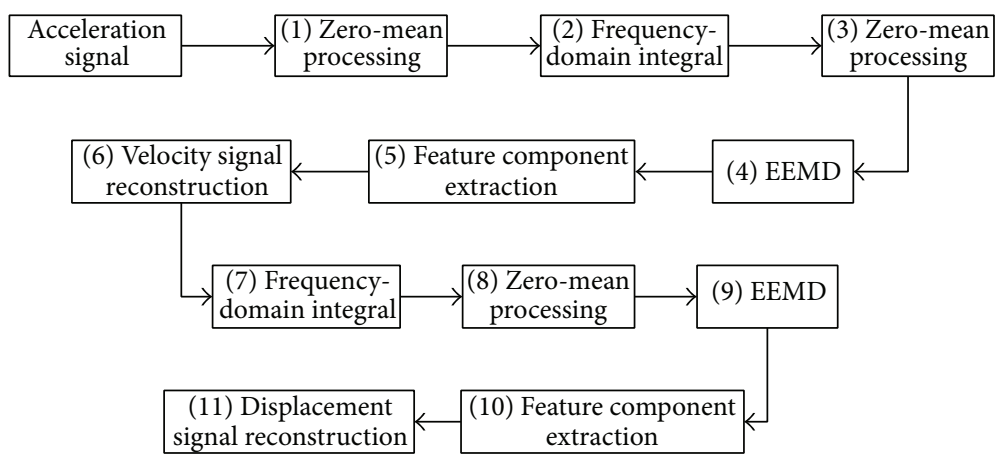

FIGURE 1: Process of the FIE-based integral method.

number alter the decomposition little as long as the added noise has moderate amplitude and the ensemble number is large enough [38]. And they suggested the empirical setting is as follows: the amplitude of the added white noise is approximately 0.2 of a standard deviation of the original signal and the number of ensemble is a few hundreds. Researchers also investigated the EEMD parameter setting for different applications $[39,40]$ and their findings are similar to that of $\mathrm{Wu}$ and Huang. Following many simulations and empirical strategy [41] from predecessors' researches, we determine the amplitude is 0.15 and the ensemble number is 100 .

(5) Feature Component Extraction for the First Time. Then, the feature vectors of each IMF obtained by step (4) are calculated as $\mathbf{T}_{i}=\left[\begin{array}{llll}a_{i} & b_{i} & c_{i} & d_{i}\end{array}\right]$, and that of the velocity signal obtained by step (3) is calculated as $\mathbf{T}_{1}=\left[\begin{array}{llll}a_{1} & b_{1} & c_{1} & d_{1}\end{array}\right]$. Furthermore, the Euclidean distance between them is calculated as $D_{i}$. Finally, the IMF that is closest to $\mathbf{T}_{1}$ is selected. In this case, the cross-correlation degree between the extracted IMF component and $\mathbf{T}_{1}$ is the largest. Consider

$$
D_{i}=\sqrt{\left(a_{1}-a_{i}\right)^{2}+\left(b_{1}-b_{i}\right)^{2}+\left(c_{1}-c_{i}\right)^{2}+\left(d_{1}-d_{i}\right)^{2}} .
$$

(6) Velocity Signal Reconstruction. Reconstruct the velocity signal according to the feature components extracted in step (5).

(7) Frequency-Domain Integral for the Second Time. From the previous researches $[20,35]$, it can be seen that a large accumulated error will be produced in TDI, and the result is largely influenced by the accumulated amplification effect of tiny error. However, the FDI can effectively avoid the amplification effect of accumulated error [1, 2]. Based on this idea, in order to overcome the flaws of traditional TDI, frequency-frequency domain combined integration method is introduced when calculating the displacement signal.

In the implementation process, FDI is utilized to integrate the reconstructed velocity signal obtained by step (6). Then, the displacement time-domain signal can be derived.

(8) Zero-Mean Processing for the Third Time. Process the displacement time-domain signal by means of zero-mean processing method, which can get rid of the direct current component mixed in the displacement signal. The purpose of this step is to eliminate the influence of trend item on the subsequent processing results.

(9) EEMD for the Second Time. The same as in step (4), EEMD is utilized to decompose the displacement signal obtained by step (8); then several IMF components which reflect different vibration modes are acquired.

(10) Feature Component Extraction for the Second Time. In this step, the feature vector of each IMF component obtained by step (9) is calculated as $\mathbf{T}_{i}=\left[\begin{array}{llll}a_{i} & b_{i} & c_{i} & d_{i}\end{array}\right]$, and that of displacement signal obtained by step (8) is calculated as $\mathbf{T}_{2}=$ $\left[\begin{array}{llll}a_{2} & b_{2} & c_{2} & d_{2}\end{array}\right]$. Moreover, the Euclidean distance between them can be calculated by (11). Finally, search and extract the specific IMF component which is closest to $\mathbf{T}_{2}$. In this case, the cross-correlation degree between the extracted IMF component and $\mathbf{T}_{2}$ is the largest.

(11) Displacement Signal Reconstruction. Reconstruct the displacement signal according to the feature components extracted in the step (10).

4.2. Indices for Error Evaluation. The correlation degree between the reconstructed signal and the theoretical signal can be seen intuitively from the waveform graph. However, in order to quantitatively evaluate the calculation accuracy of different methods, it is necessary to search proper evaluation indices. Based on the previous study $[17,35]$, this paper takes the average peak error, the average maximum relative error, and the error of square sum as evaluation indices.

The average peak error presents the correlation degree of waveform maximum range between integral signal and theoretical signal, which is expressed as follows:

$$
\begin{aligned}
E_{\mathrm{rp}}=\frac{1}{2}\left\{\frac{\max [X(t)]-\max [S(t)]}{\max [S(t)]}\right. \\
\left.+\frac{\min [X(t)]-\min [S(t)]}{\min [S(t)]}\right\} .
\end{aligned}
$$

The average maximum relative error presents the local maximum error of the integral signal. Through this index, the maximum error of the integral signal relative to the 
theoretical signal can be known, which is represented as follows:

$$
E_{\mathrm{rr}}=\frac{1}{2}\left\{\frac{\max [X(t)-S(t)]}{\max [S(t)]}+\frac{\min [X(t)-S(t)]}{\min [S(t)]}\right\} .
$$

When comparing and evaluating two waveforms, their overall effect which means the difference in representing energy of waveform also should be investigated. The error of square sum can describe the energy error between the integral signal and the corresponding theoretical signal, which is depicted as follows:

$$
E_{\mathrm{rsq}}=\frac{\sum_{i=1}^{N}[X(i)]^{2}-\sum_{i=1}^{N}[S(i)]^{2}}{\sum_{i=1}^{N}[S(i)]^{2}} .
$$

In formulas (12)-(14), $X(t)$ denotes the sampling value of the signal obtained by integration, $S(t)$ is the sampling value of the theoretical signal, and $N$ is the number of the sampling points.

\section{Simulation Analysis}

The simulation signal imitates a vibration situation of rotating machinery under complex coupling fault. The rotational frequency is $30 \mathrm{~Hz}$. In addition to self-excited vibration, the rotating machinery is also accompanied by rubbing fault between moving and stationary parts. It results in a complex signal structure which consists of many components in the acceleration signal spectrum, such as 0.2 subharmonic frequency, onefold frequency, twofold frequency, and threefold frequency. The form of the simulation signal is as follows:

$$
\begin{aligned}
a(t)= & 0.05 \cos \left(2 \pi \times 6 t+130 \times \frac{\pi}{180}\right) \\
& +0.25 \cos \left(2 \pi \times 30 t+18 \times \frac{\pi}{180}\right) \\
& +0.15 \cos \left(2 \pi \times 60 t+43 \times \frac{\pi}{180}\right) \\
& +0.066 \cos \left(2 \pi \times 90 t+77 \times \frac{\pi}{180}\right) \\
& +0.028 \cos \left(2 \pi \times 120 t+118 \times \frac{\pi}{180}\right)+0.01 .
\end{aligned}
$$

According to the Shannon sampling theorem, the sampling frequency is taken as $1024 \mathrm{~Hz}$. The sampling number is taken as 1024 . In order to represent the real situation, the Gaussian white noise is added into the simulated signal at the ratio $\mathrm{SNR}=10$. The simulated signals and their amplitude spectra are displayed in Figure 2.

In this section, three integral methods are adopted to analyze the simulated signal. They are TDI-, FDI-, and the FIE-based integral method. Furthermore, the errors of results are compared. The detailed processes are as follows.

(1) Analysis for Time-Domain Integral. The original acceleration signal is firstly processed by means of zero-mean processing to get rid of the direct current component. And

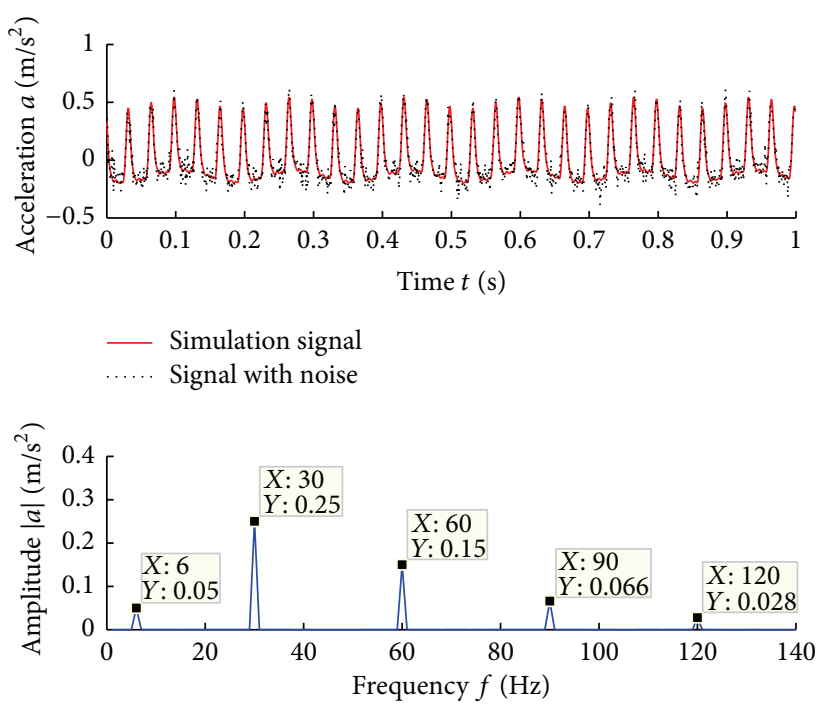

Figure 2: Simulated signals and their amplitude spectra.
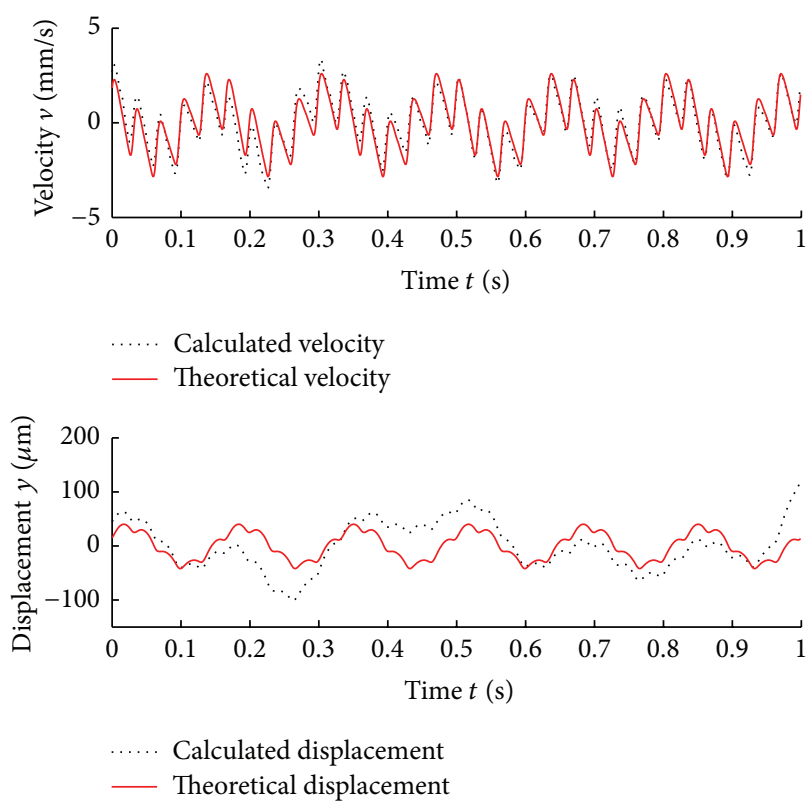

FIGURE 3: Comparison of the TDI results with the theoretical signals.

the trend item of velocity and displacement signal obtained by TDI is eliminated through least squares fitting. The comparison between the integral results and the theoretical signals is depicted in Figure 3.

Figure 3 indicates that the low-frequency trend item of TDI in the first time is commendably eliminated through zero-mean processing and least squares fitting. However, because the effect of noise cannot be removed, the integral results still have considerable errors. With the increase in integral times, the errors gradually increase. The results of TDI in the second time produce obvious distortion.

(2) Analysis for Frequency-Domain Integral. In order to reduce the effect of low-frequency noise and trend item, the 
frequency components lower than $3 \mathrm{~Hz}$ are set to zero. The comparison between the integral results and the theoretical signals is described in Figure 4.

Figures 3 and 4 suggest that, if only comparing the timedomain waveforms, the results of FDI are obviously better than those of TDI. By comparing the results of FDI and the theoretical signals, it can be found that they have a higher fitting degree. However, Figure 4 also indicates that set-tozero processing only can effectively remove the trend item of FDI in the first time. The influence of the trend item still exists and the results of FDI in the second time still have obvious errors.

(3) Analysis for FIE-Based Integral. Now, the acceleration signal is analyzed with the method proposed in this paper. The comparison between the integral results and the theoretical signals is displayed in Figure 5.

Compared with traditional TDI and FDI, the results of FIE-based integral are the best. They show the best fitting degree with the theoretical signal.

The quantitative error evaluation results and comparisons of the three integral methods are shown in Tables 1 and 2, respectively.

From Tables 1 and 2, it can be seen that the integral accuracy of FIE-based approach is better than TDI and FDI. With the increase in integration times, the errors will not get larger. The result indicates that the proposed method has a better accuracy and reliability when it comes to vibration signal integration of rotating machinery. And the integral results have the minimum distortion caused by interference factors such as noise and trend item.

\section{Experiment Data Analysis}

As mentioned above, for faulty rotating machinery, the effective components of vibration signals mainly consist of rotational frequency and fault characteristic frequency. In the signal spectrum, the frequency characteristic where spectrum peaks appear can be used to estimate fault type. Moreover, these characteristics can be further utilized to determine whether the signal effectively contains useful feature information. In order to further validate the effectiveness of FIE-based integral approach, the rolling bearing commonly used in the rotating machinery is taken as the research object. Furthermore, the practicability of the proposed method is further verified via analyzing the measured vibration signals and the acquired integration signals.

The fault characteristic frequency expression of the bearing outer race is [42]

$$
f_{o}=\frac{z}{2}\left(1-\frac{d_{0}}{D} \cos \alpha\right) f_{r}
$$

The fault characteristic frequency expression of the bearing inner race is

$$
f_{i}=\frac{z}{2}\left(1+\frac{d_{0}}{D} \cos \alpha\right) f_{r}
$$
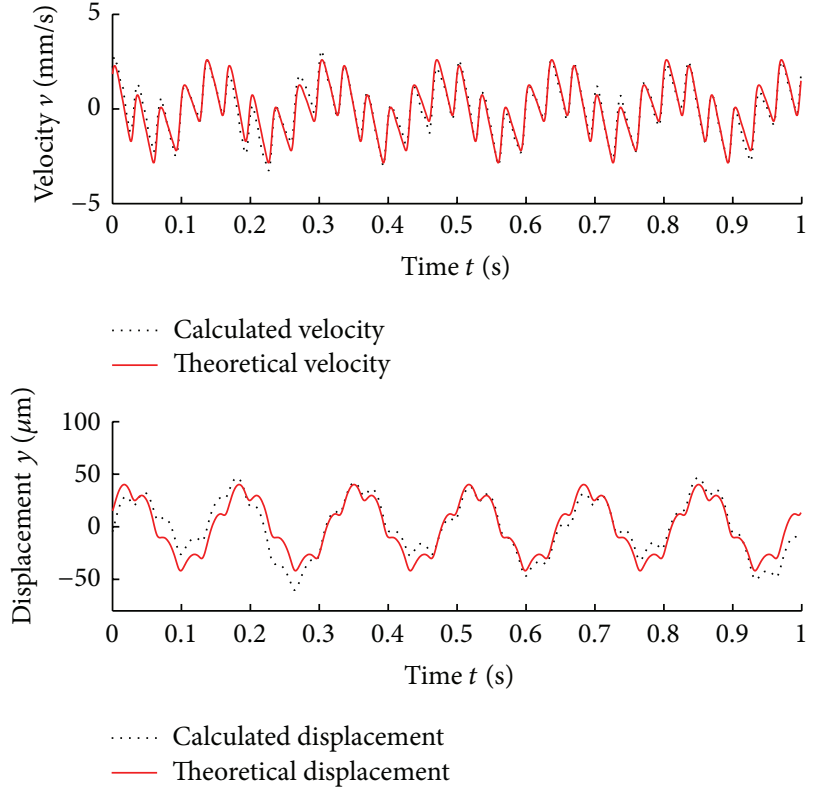

FIGURE 4: Comparison of the FDI results with the theoretical signals.
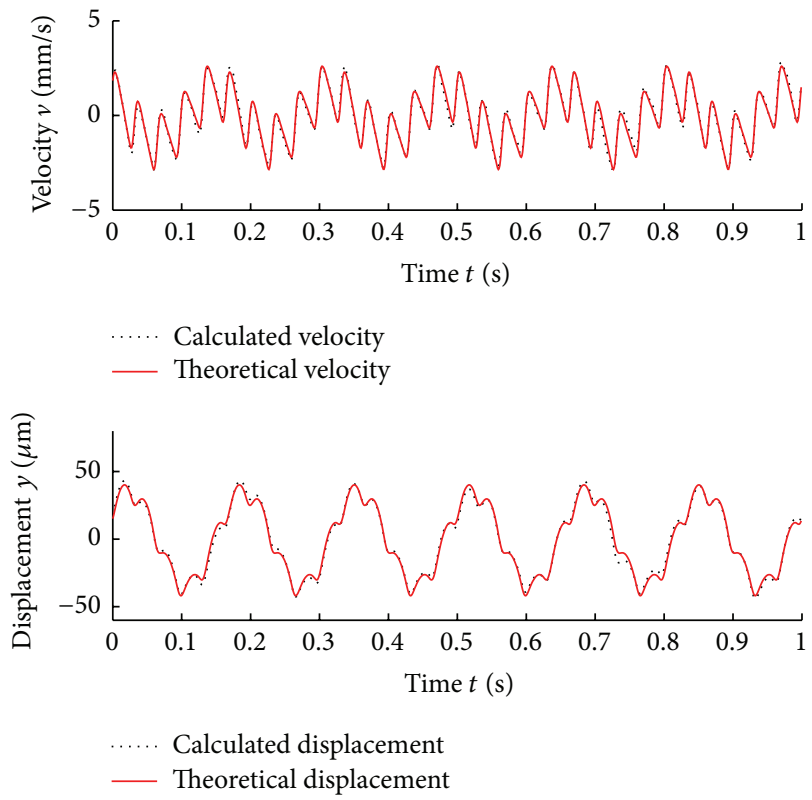

FIGURE 5: Comparison of FIE-based results with the theoretical signals.

where $z$ is the number of balls, $d_{0}$ is the ball diameter, $D$ is the pitch diameter of the bearing raceway, $\alpha$ is the pressure angle, $f_{r}$ is the shaft rotational frequency, $f_{r}=n / 60$, and $n$ is the shaft rotation speed.

This paper adopts the experiment data measured in the fault test bench of rolling bearing from Case Western Reserve University in America. As shown in Figure 6, the test bench consists of a motor (left), a torque transducer (center), a dynamometer (right), and electronic control device (not shown) [43]. The motor shaft is supported by the test bearings 
TABLE 1: Error comparison of integral velocity.

\begin{tabular}{lccc}
\hline Integral method & Average peak error & $\begin{array}{c}\text { Error indices } \\
\text { Average maximum relative error }\end{array}$ & $\begin{array}{c}\text { Error of square sum } \\
E_{\mathrm{rsq}}\end{array}$ \\
\hline TDI & $E_{\mathrm{rp}}$ & 0.4200 & 0.0786 \\
FDI & 0.2475 & 0.2988 & 0.0211 \\
FIE & 0.1681 & 0.1797 & 0.0208 \\
\hline
\end{tabular}

TABLE 2: Error comparison of integral displacement.

\begin{tabular}{|c|c|c|c|}
\hline \multirow[b]{2}{*}{ Integral method } & \multicolumn{3}{|c|}{ Error indices } \\
\hline & $\begin{array}{c}\text { Average peak error } \\
E_{\mathrm{rp}}\end{array}$ & $\begin{array}{l}\text { Average maximum relative error } \\
\qquad E_{\mathrm{rr}}\end{array}$ & $\begin{array}{c}\text { Error of square sum } \\
\qquad E_{\mathrm{rsq}}\end{array}$ \\
\hline TDI & 1.7352 & 2.2435 & 2.0133 \\
\hline FDI & 0.2996 & 0.5452 & 0.1030 \\
\hline FIE & 0.0661 & 0.1476 & 0.0077 \\
\hline
\end{tabular}

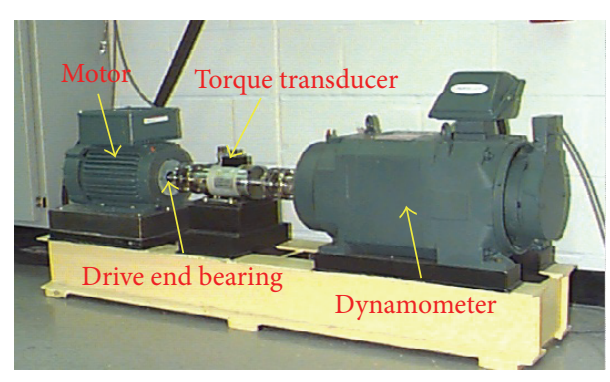

FiguRE 6: Fault test bench of rolling bearing.

whose type is 6025-2RS JEM SKF. The detailed parameters of the bearings are displayed in Table 3.

In the experiment, single point faults are introduced to the test bearings using electrodischarge machining with different fault diameters. Faults ranging from $0.1778 \mathrm{~mm}$ to $1.016 \mathrm{~mm}$ in diameter are introduced separately at the inner raceway, outer raceway, and rolling element. Faulted bearings were reinstalled into the test motor and vibration data was recorded for motor loads of 0 to 3 horsepower (motor speeds of 1797 to $1720 \mathrm{rpm}$ ). Vibration data is collected using accelerometers, which are attached to the housing with magnetic bases. Accelerometers are placed at the 12 oclock position at both drive end and fan end of the motor housing. Vibration signals are collected using a 16-channel DAT recorder and are postprocessed in a Matlab environment. Digital data is collected at 12,000 samples per second for drive end bearing faults. Speed and horsepower data are collected using the torque transducer.

This paper takes fault data of drive end bearings as samples. The diameter of the damage points is $0.1778 \mathrm{~mm}$ and the depth is $0.2794 \mathrm{~mm}$. The sampling frequency is $12 \mathrm{kHz}$. The sampling number is 10240 . According to (16), (17), and Table 3, when the motor rotation speed is $1797 \mathrm{rpm}$, the shaft rotational frequency is about $30 \mathrm{~Hz}$ and the fault
TABLE 3: Parameters of the 6205-2RS JEM SKF bearing.

\begin{tabular}{|c|c|c|c|}
\hline $\begin{array}{l}\text { Pitch diameter } \\
D\end{array}$ & $\begin{array}{l}\text { Ball diameter } \\
\qquad d_{0}\end{array}$ & $\begin{array}{l}\text { Number of balls } \\
z\end{array}$ & $\begin{array}{l}\text { Pressure angle } \\
\qquad \alpha \\
\end{array}$ \\
\hline $39.04 \mathrm{~mm}$ & $7.94 \mathrm{~mm}$ & 9 & $0^{\circ}$ \\
\hline
\end{tabular}

characteristic frequencies of the outer and inner race are about $107 \mathrm{~Hz}$ and $162 \mathrm{~Hz}$.

The time-domain waveforms of the vibration acceleration signals are shown in Figure 7. The signals are measured in the state of normal, outer race fault and inner race fault. It is hard to recognize the pulse component associated with the faults from the time-domain graphs because the signals are mixed with intense noise. In order to further compare the effectiveness among the proposed FIE-based integral approach and traditional integral methods, when implementing traditional integral methods, the original signals are preprocessed with widely used wavelet denoising (softthresholding-based). Moreover, in TDI, eliminating direct current is performed and the integral results are amended with the popular least squares fitting. In FDI, the set-to-zero processing method is utilized to remove the interferences of trend item. Then, the obtained signals are converted into frequency domain and the fault types can be determined through identifying the characteristic frequencies.

The amplitude spectra of vibration acceleration signals of the aforementioned three states after wavelet denoising are represented in Figure 8. From Figure 8(a), it can be seen that typical side band is presented in the amplitude spectrum. In order to clearly and exactly identify fault, the frequency band $20 \sim 200 \mathrm{~Hz}$ is selected as a key analysis section according to the calculated fault characteristic frequencies. The frequency range can completely contain all values of characteristic frequencies, such as $30 \mathrm{~Hz}, 107 \mathrm{~Hz}$, and $162 \mathrm{~Hz}$, as displayed in Figure 8(b). For normal signal, some peaks occur at the 


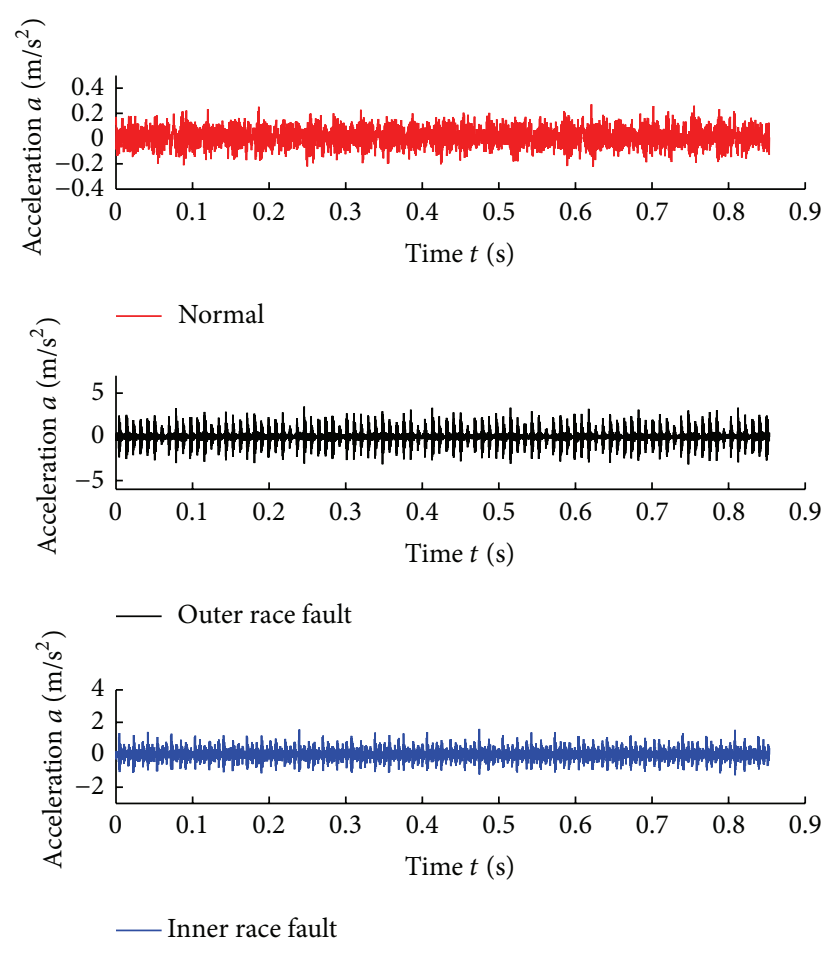

FIGURE 7: Time-domain waveform of the original acceleration signals.

rotation shaft fundamental frequency $30 \mathrm{~Hz}$ and its superharmonic in the amplitude spectrum. When it comes to outer race fault, the peak occurs at the fault characteristic frequency $107 \mathrm{~Hz}$. And for inner race fault, it occurs at $162 \mathrm{~Hz}$. These indicate that the signals after wavelet denoising effectively include fault information. The fault types can be visually determined from the amplitude spectra of the denoised signals. However, Figure 8 also demonstrates that the noise in the signals is too intense to be completely eliminated, which makes some clutter waves appear in the amplitude spectrum of the normal signal. Moreover, the characteristic frequencies at the fundamental frequency $30 \mathrm{~Hz}$ and its superharmonic are almost drowned in the amplitude spectra of the outer and inner race fault signals.

The results performed by traditional integral methods are portrayed in Figures 9 and 10. They indicate that the time-domain first integral commendably reduces the interference from the trend item after direct current elimination, wavelet denoising, and least squares fitting. The characteristic frequencies in the amplitude spectra of the velocity signals are well presented. However, the trend item in acceleration signals cannot be completely eliminated, and the produced cumulative errors greatly affect the calculation accuracy. Specifically after the second integration, the displacement amplitudes have a large deviation. Moreover, the influence of noise still exists as it cannot be completely eliminated. The characteristic frequency of outer race fault is almost drowned in the amplitude spectrum of the displacement signal, which leads to the failure to effectively determine the fault types.

Meanwhile, Figures 9 and 10 also reveal that FDI is still under the influences of low-frequency noise and trend item after wavelet denoising and set-to-zero processing. The velocity and displacement amplitudes have a large deviation after integration. In comparison, the influence of noise on the first integration is relatively small. The characteristic frequencies in the amplitude spectra of the velocity signals are well presented. Nevertheless, the second integral undergoes an intense influence of noise. The characteristic frequencies are almost drowned in the amplitude spectra of the displacement signals, which makes the fault types failure to be effectively determined.

Now, without any preprocessing technology, the original acceleration signals are performed with the proposed FIEbased method. Then the same as the aforementioned discussion, we analyze the results in the frequency domain. The amplitude spectra of the velocity and displacement signals after integration are depicted in Figures 11 and 12.

Figures 11 and 12 indicate that clutter waves in the amplitude spectra of the normal signals are effectively restrained. The background noise of the fault signals is also effectively restrained. Moreover, the characteristic frequencies at the fundamental frequency $30 \mathrm{~Hz}$ and its superharmonic are commendably presented. The fault types can be effectively determined because the fault characteristic frequencies are clearly represented. The results reveal that both velocity and displacement signals, which are obtained by FIE-based method, effectively include the useful fault information. And the connotative feature information is accurately extracted from the complex vibration signal. The effectiveness and practicability of the proposed method are successfully verified. 

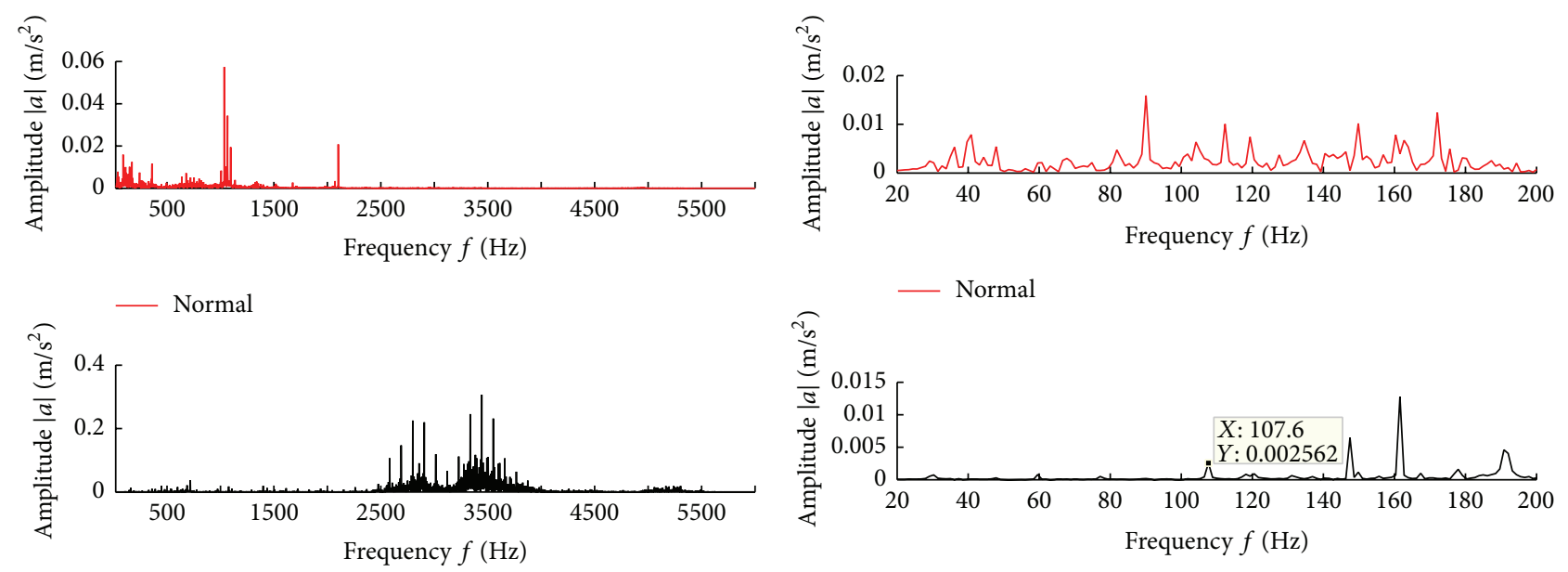

- Outer race fault

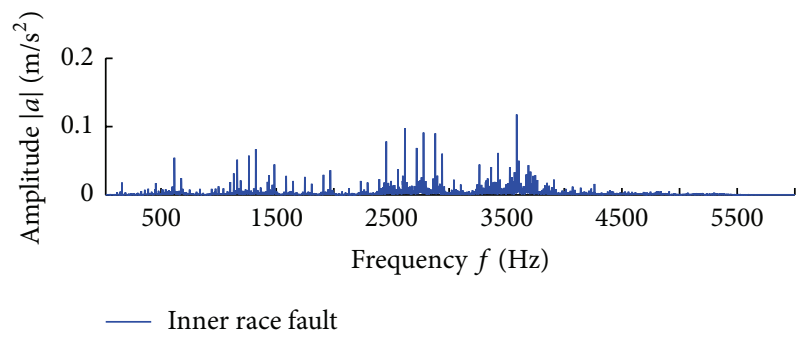

(a) Amplitude spectra

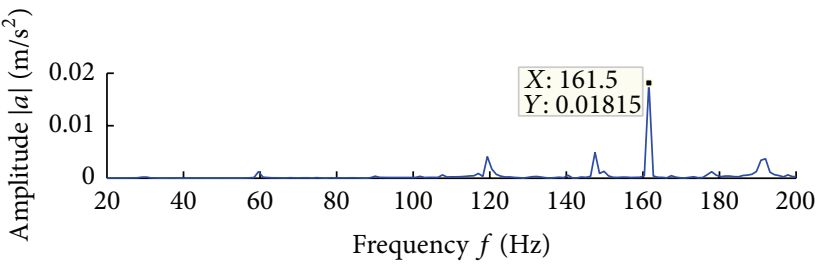

— Inner race fault

(b) Local amplitude spectra

FIGURE 8: Amplitude spectra of the acceleration signals after wavelet denoising processing.
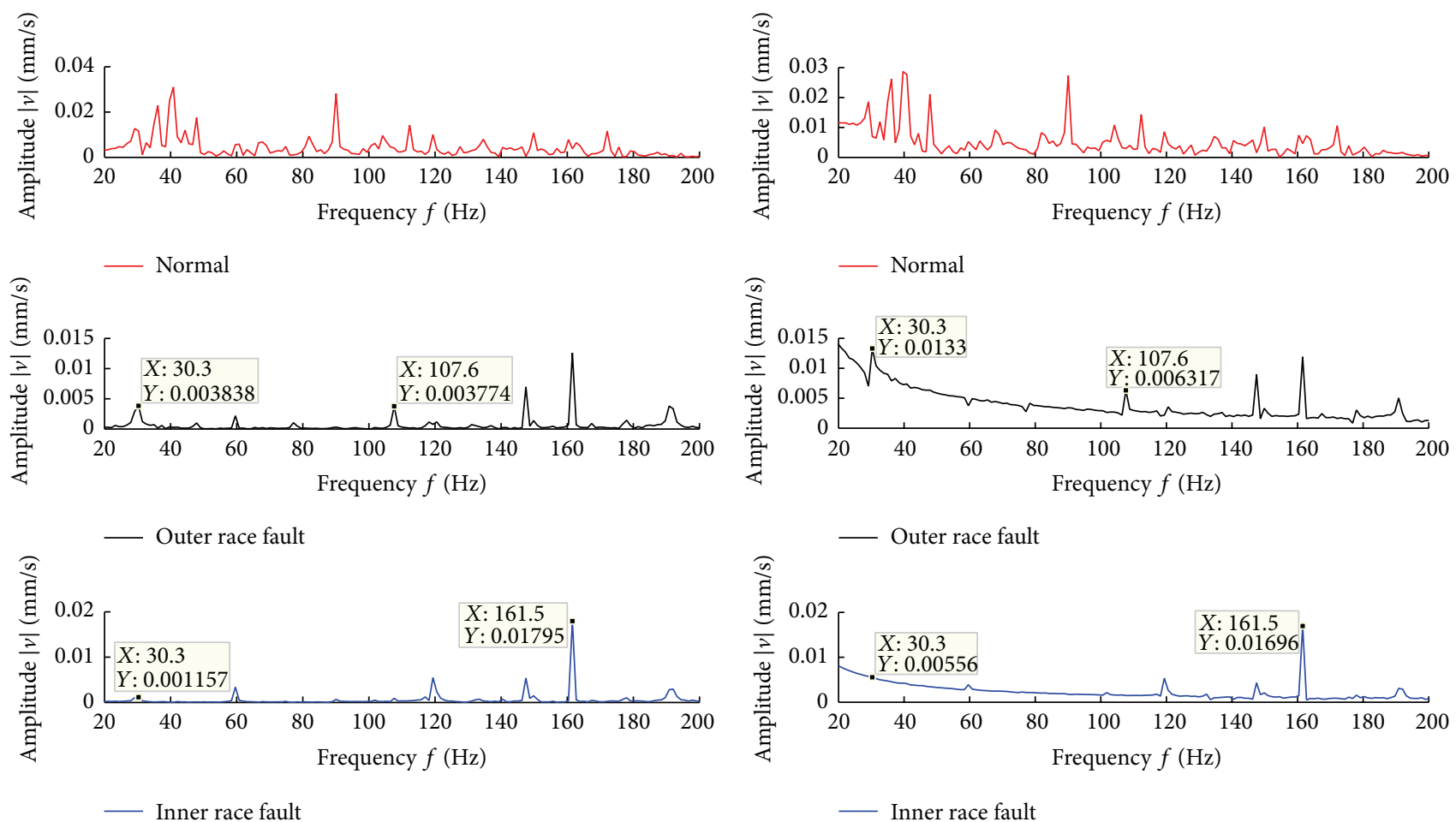

(a) Time-domain first integral

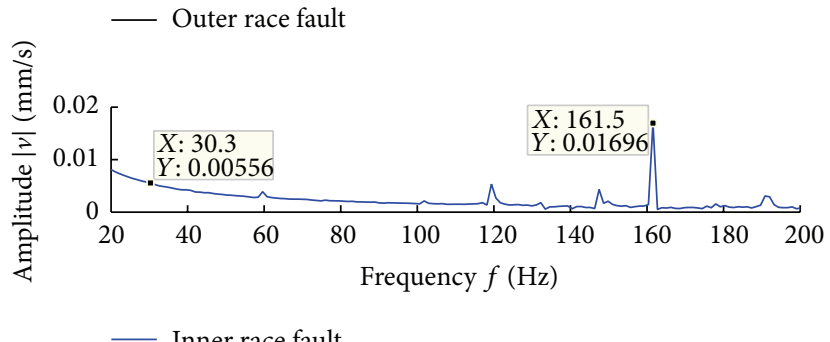

(b) Frequency-domain first integral

FIGURE 9: Amplitude spectra of the velocity signals acquired by traditional integral. 

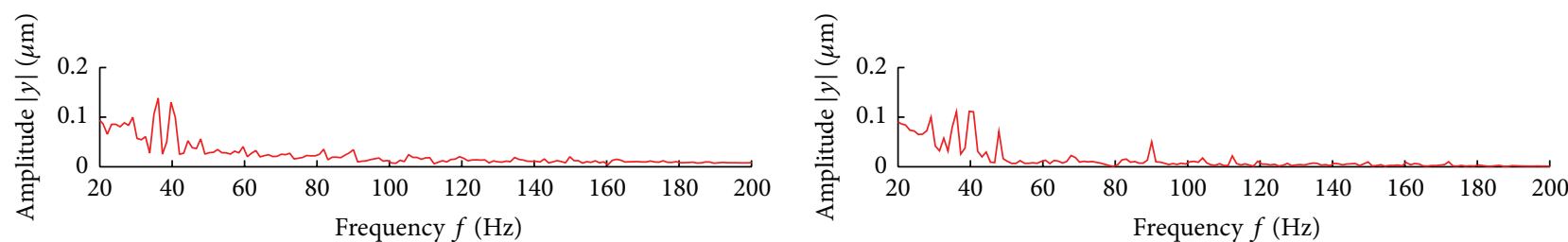

_ Normal

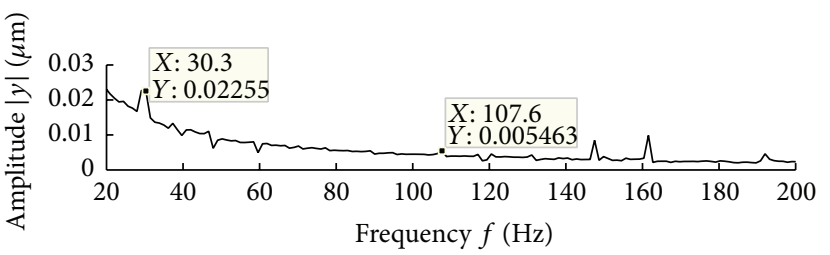

— Normal

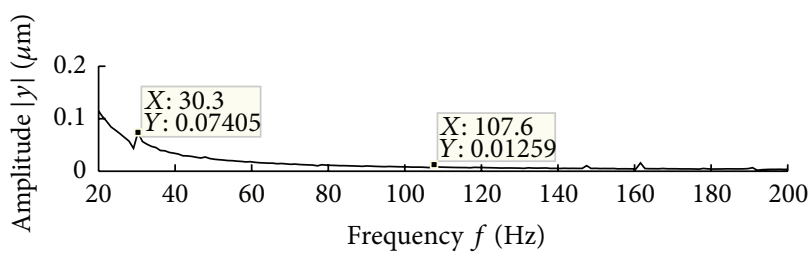

_ Outer race fault

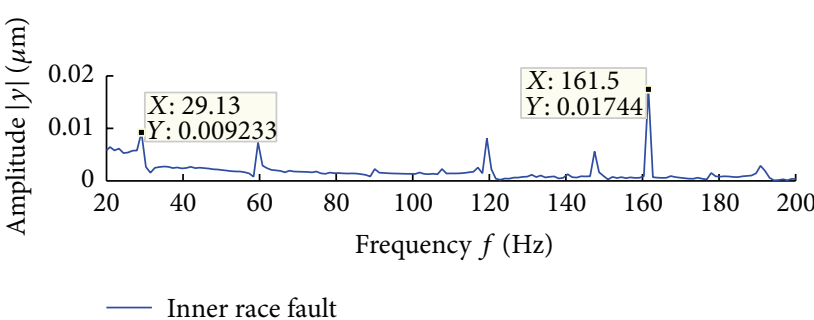

(a) Time-domain second integral

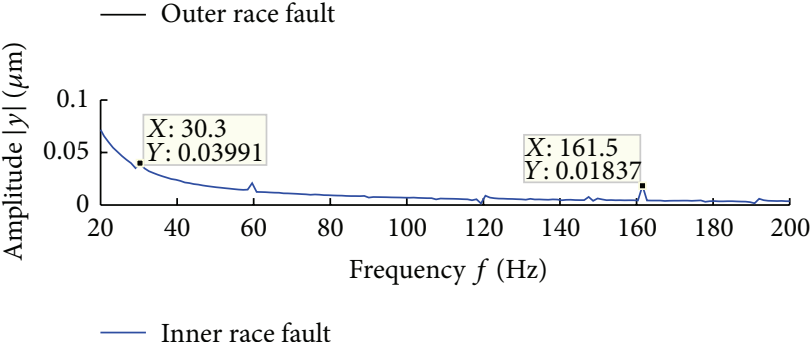

(b) Frequency-domain second integral

FIGURE 10: Amplitude spectra of the displacement signals acquired by traditional integral.

$$
\begin{aligned}
& \text { क् } \\
& \text { है } \\
& \frac{2}{2} \\
& \frac{0}{0} \\
& \text { : } \\
& \text { है }
\end{aligned}
$$

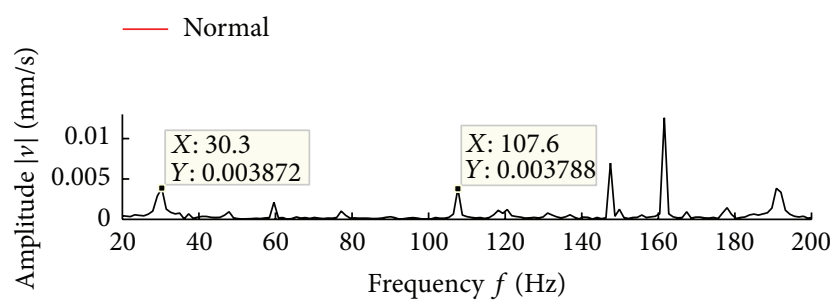

_ Outer race fault

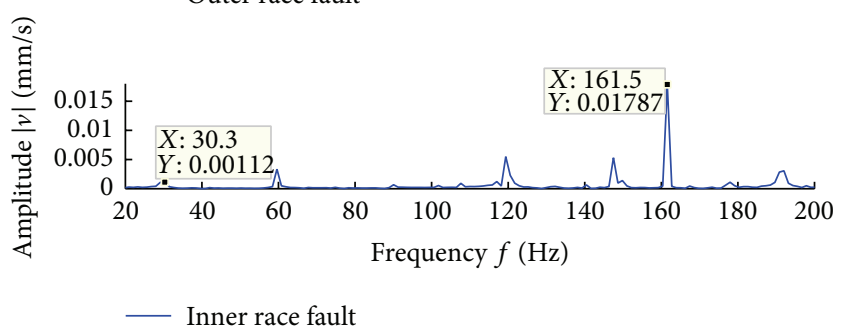

FIGURE 11: Amplitude spectra of the velocity signals acquired by FIE.

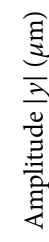

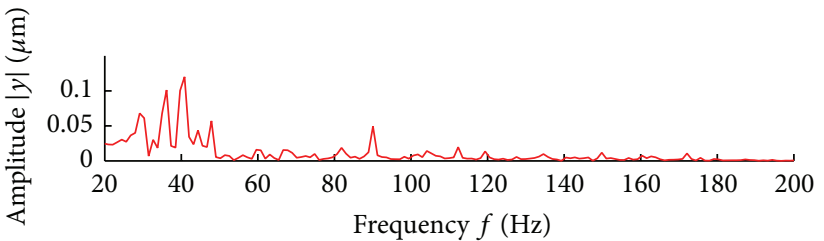

— Normal

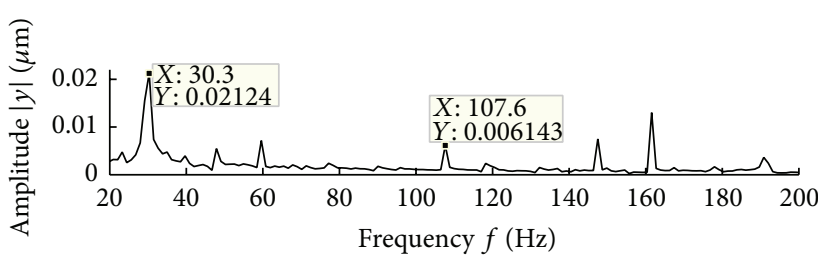

_ Outer race fault

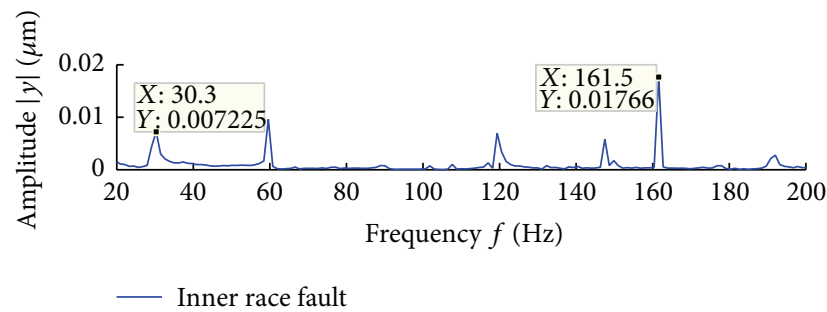

FIGURE 12: Amplitude spectra of the displacement signals acquired by FIE. 


\section{Conclusions}

This paper makes a thorough research on the integral method of vibration signal through theoretical analysis, simulation studies, and the measured data verification. We can draw some conclusions from our study.

(1) The proposed FIE-based integral method takes full advantage of the self-adaptive filter characteristic and waveform correction feature of EEMD in dealing with nonlinear and nonstationary signals. Furthermore, this research merges the superiorities of kurtosis, mean square error, energy, and singular value decomposition on signal feature extraction. The desired signals are precisely obtained.

(2) With the proposed method, the interference problem of invalid signal such as trend item and noise which plague traditional integral methods is commendably solved. The great cumulative error from the traditional time-domain integral is effectively overcome. Moreover, the large low-frequency error from the traditional frequency-domain integral is successfully avoided. Comparing with the traditional integral, this method is outstanding at removing noise and retaining useful feature information and shows higher accuracy and superiority.

(3) The proposed method takes full consideration of the structural characteristics of vibration signal. It is facile to implement and possesses considerable practicability. This research can provide important vibration parameters and basis for the follow-up study on equipment fault diagnosis and condition monitoring.

\section{Conflict of Interests}

The authors declare that there is no conflict of interests regarding the publication of this paper.

\section{Acknowledgments}

This work is supported by the National Key Basic Research Program (973 Program) of China (2014CB046405), National Natural Science Foundation of China (51475405), and Natural Science Foundation of Hebei Province, China (E2013203161, E2013203114). The support is gratefully acknowledged. The authors would also like to thank the reviewers for their valuable suggestions and comments.

\section{References}

[1] Q. Li, T. Y. Wang, and Y. G. Xu, "The modification of the vibration parameter transform based on frequency domain integration," Modular Machine Tool \& Automatic Manufacturing Technique, vol. 9, pp. 60-61, 65, 2005.

[2] M. K. Gu and Z. H. Lü, "Identification of a mechanism's vibration velocity and displacement based on the vibration acceleration measurement," Mechanical Science and Technology for Aerospace Engineering, vol. 30, pp. 522-526, 2011.
[3] L. Dong, M. F. Liao, and S. J. Yang, "Filtering modification of vibration signal integration algorism," Machinery Design \& Manufacture, vol. 1, pp. 46-48, 2010.

[4] X. H. Fan, Z. Y. Zhao, G. An, and K. Wang, "Research on the arithmetic of mechanical vibration severity in frequency domain," Journal of Academy of Armored Force Engineering, vol. 22, no. 1, pp. 42-45, 2008.

[5] L. Fu and X. K. Tang, "Improving analytical precisions of software used in condition monitoring systems," Journal of Tsinghua University, vol. 38, no. 8, pp. 111-113, 1998.

[6] Y. K. Thong, M. S. Woolfson, J. A. Crowe, B. R. Hayes-Gill, and R. E. Challis, "Dependence of inertial measurements of distance on accelerometer noise," Measurement Science and Technology, vol. 13, no. 8, pp. 1163-1172, 2002.

[7] Y. K. Thong, M. S. Woolfson, J. A. Crowe, B. R. Hayes-Gill, and D. A. Jones, "Numerical double integration of acceleration measurements in noise," Measurement: Journal of the International Measurement Confederation, vol. 36, no. 1, pp. 73-92, 2004.

[8] J. Yang, J. B. Li, and G. Lin, "A simple approach to integration of acceleration data for dynamic soil-structure interaction analysis," Soil Dynamics and Earthquake Engineering, vol. 26, no. 8, pp. 725-734, 2006.

[9] X. X. Zhou, E. K. Chen, and G. Q. Lü, “The processing of vibration acceleration signal based on numeric integration and LMS," Process Automation Instrumentation, vol. 27, pp. 51-53, 2006.

[10] Y. Zhang, J. Song, L. Tu, and S. Xue, "Error analysis and improvement method when numerical integration with software," Journal of Mechanical Strength, vol. 28, no. 3, pp. 419-423, 2006.

[11] Y. H. Hong, H. W. Park, and H. S. Lee, "A regularization scheme for displacement reconstruction using acceleration data measured from structures," in Sensors and Smart Structures Technologies for Civil, Mechanical, and Aerospace Systems, vol. 6932 of Proceedings of SPIE, San Diego, Calif, USA, 2008.

[12] S. Han and J. B. Lee, "Analysis of errors in the conversion of acceleration into displacement," in Proceedings of 19th International Modal Analysis Conference (IMAC XIX '01), pp. 14081413, 2001.

[13] S. Han and J. W. Chung, "Retrieving displacement signal from measured acceleration signal," in Proceedings of 20th International Modal Analysis Conference (IMAC '02), pp. 11781184, 2002.

[14] S. Han, "Retrieving the time history of displacement from measured acceleration signal," KSME International Journal, vol. 17, no. 2, pp. 197-206, 2003.

[15] J. G. T. Ribeiro, J. T. P. Castro, and J. L. F. Freire, "Using the FFT-DDI method to measure displacement with piezoelectric, resistive and ICP accelerometers," in Proceedings of the 22nd IMAC Conference \& Exposition on Structural Dynamics (IMACXXI '03), Kissimee, Fla, USA, 2003.

[16] Z. Y. Duan, P. M. Jia, Y. F. Xu, and J. Z. Hu, "Research \& application of software integration of vibration fault signal," Machine Building \& Automation, vol. 36, pp. 76-78, 81, 2007.

[17] G. Wen, Y. Li, Y. Liao, and Q. He, "Faulty rotor system vibration acceleration signal integration method based on precise information reconstruction," Journal of Mechanical Engineering, vol. 49, no. 8, pp. 1-9, 2013.

[18] H.-V. Liew and T. C. Lim, "Analysis of time-varying rolling element bearing characteristics," Journal of Sound and Vibration, vol. 283, no. 3-5, pp. 1163-1179, 2005. 
[19] T. R. Kurfess, S. Billington, and S. Liang, "Advanced diagnostic and prognostic techniques for rolling element bearings," in Condition Monitoring and Control for Intelligent Manufacturing, Springer Series in Advanced Manufacturing, pp. 137-165, Springer, London, UK, 2006.

[20] G. K. Sharma, A. Kumar, C. Babu Rao, T. Jayakumar, and B. Raj, "Short time Fourier transform analysis for understanding frequency dependent attenuation in austenitic stainless steel," NDT and E International, vol. 53, pp. 1-7, 2013.

[21] X.-J. Yang, D. Baleanu, and J. A. Tenreiro MacHado, "Mathematical aspects of the Heisenberg uncertainty principle within local fractional Fourier analysis," Boundary Value Problems, vol. 2013, article 131, 16 pages, 2013.

[22] P. S. Addison, The Illustrated Wavelet Transform Handbook: Introductory Theory and Applications in Science, Engineering, Medicine and Finance, CRC Press, New York, NY, USA, 2010.

[23] L. J. Meng, J. W. Xiang, Y. X. Wang, Y. Y. Jiang, and H. F. Gao, "A hybrid fault diagnosis method using morphological filter-translation invariant wavelet and improved ensemble empirical mode decomposition," Mechanical Systems and Signal Processing, vol. 50-51, pp. 101-115, 2015.

[24] L. Stanković, I. Djurović, and R.-M. Laković, "Instantaneous frequency estimation by using the Wigner distribution and linear interpolation," Signal Processing, vol. 83, no. 3, pp. 483491, 2003.

[25] G. Chen, J. Chen, G. M. Dong, and H. M. Jiang, "An adaptive non-parametric short-time Fourier transform: application to echolocation," Applied Acoustics, vol. 87, pp. 131-141, 2015.

[26] Z. Wu and N. E. Huang, "Ensemble empirical mode decomposition: a noise-assisted data analysis method," Advances in Adaptive Data Analysis, vol. 1, no. 1, pp. 1-41, 2009.

[27] N. E. Huang, Z. Shen, S. R. Long et al., "The empirical mode decomposition and the Hilbert spectrum for nonlinear and non-stationary time series analysis," The Royal Society of London. Proceedings Series A: Mathematical, Physical and Engineering Sciences, vol. 454, no. 1971, pp. 903-995, 1998.

[28] M. Amarnath and I. R. P. Krishna, "Detection and diagnosis of surface wear failure in a spur geared system using EEMD based vibration signalanalysis," Tribology International, vol. 61, pp. 224-234, 2013.

[29] W. Guo and P. W. Tse, "A novel signal compression method based on optimal ensemble empirical mode decomposition for bearing vibration signals," Journal of Sound and Vibration, vol. 332, no. 2, pp. 423-441, 2013.

[30] J. F. Wang, J. Ma, R. G. Ma, and H. X. Song, "Study on calculation of dynamic displacement from time-frequency integration of acceleration," Computer Science, vol. 37, pp. 201-202, 2010.

[31] B. Liang, S. D. Iwnicki, and Y. Zhao, "Application of power spectrum, cepstrum, higher order spectrum and neural network analyses for induction motor fault diagnosis," Mechanical Systems and Signal Processing, vol. 39, no. 1-2, pp. 342-360, 2013.

[32] S. Hussain and H. A. Gabbar, "Gearbox fault detection using real coded genetic algorithm and novel shock response spectrum features extraction," Journal of Nondestructive Evaluation, vol. 33, no. 1, pp. 111-123, 2014.

[33] G. L. McDonald, Q. Zhao, and M. J. Zuo, "Maximum correlated Kurtosis deconvolution and application on gear tooth chip fault detection," Mechanical Systems and Signal Processing, vol. 33, pp. 237-255, 2012.

[34] J. Ni and X. Chen, "Steady-state mean-square error analysis of regularized normalized subband adaptive filters," Signal Processing, vol. 93, no. 9, pp. 2648-2652, 2013.
[35] L. W. Jiang, L. K. Yao, and W. Wu, "Study on calculation of dynamic displacement from time integration of acceleration in shaking table model tests of side slope," Journal of Disaster Prevention and Mitigation Engineering, vol. 29, pp. 261-266, 2009.

[36] J. Cheng, D. Yu, J. Tang, and Y. Yang, "Application of SVM and SVD technique based on EMD to the fault diagnosis of the rotating machinery," Shock and Vibration, vol. 16, no. 1, pp. 8998, 2009.

[37] M. Kang and J.-M. Kim, "Singular value decomposition based feature extraction approaches for classifying faults of induction motors," Mechanical Systems and Signal Processing, vol. 41, no. 1-2, pp. 348-356, 2013.

[38] Y. Zhang and Z. Xie, "Ensemble empirical mode decomposition of impact-echo data for testing concrete structures," NDT and E International, vol. 51, pp. 74-84, 2012.

[39] J. Zhang, R. Yan, R. X. Gao, and Z. Feng, "Performance enhancement of ensemble empirical mode decomposition," Mechanical Systems and Signal Processing, vol. 24, no. 7, pp. 2104-2123, 2010.

[40] W. Guo, P. W. Tse, and A. Djordjevich, "Faulty bearing signal recovery from large noise using a hybrid method based on spectral kurtosis and ensemble empirical mode decomposition," Measurement: Journal of the International Measurement Confederation, vol. 45, no. 5, pp. 1308-1322, 2012.

[41] W. Guo and P. W. Tse, "Enhancing the ability of ensemble empirical mode decomposition in machine fault diagnosis," in Proceedings of the Prognostics and System Health Management Conference (PHM '10), Macau, China, January 2010.

[42] G. F. Wang, X. L. Feng, and C. Liu, "Bearing fault classification based on conditional random field," Shock and Vibration, vol. 20, no. 4, pp. 591-600, 2013.

[43] The Case Western Reserve University Bearing Data Center, "Bearing data center fault test data [EB/OL]," http://csegroups .case.edu/bearingdatacenter/home. 

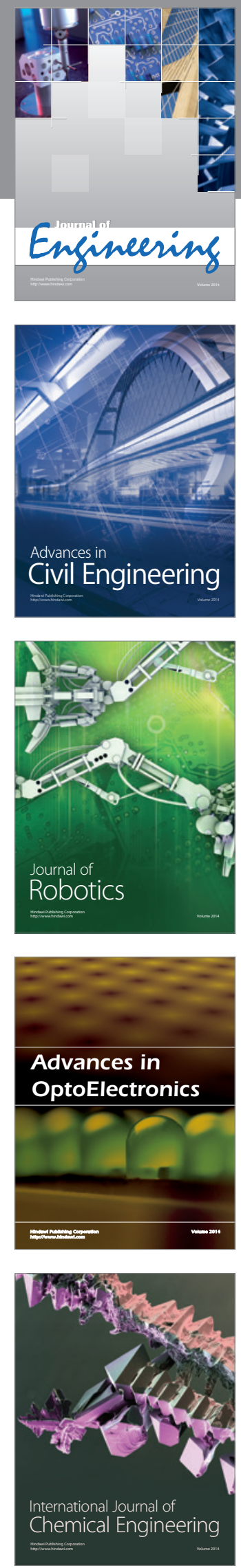

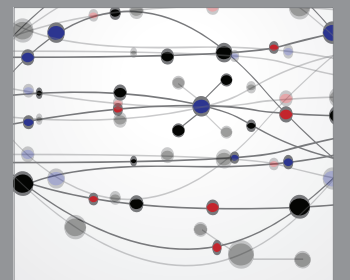

The Scientific World Journal
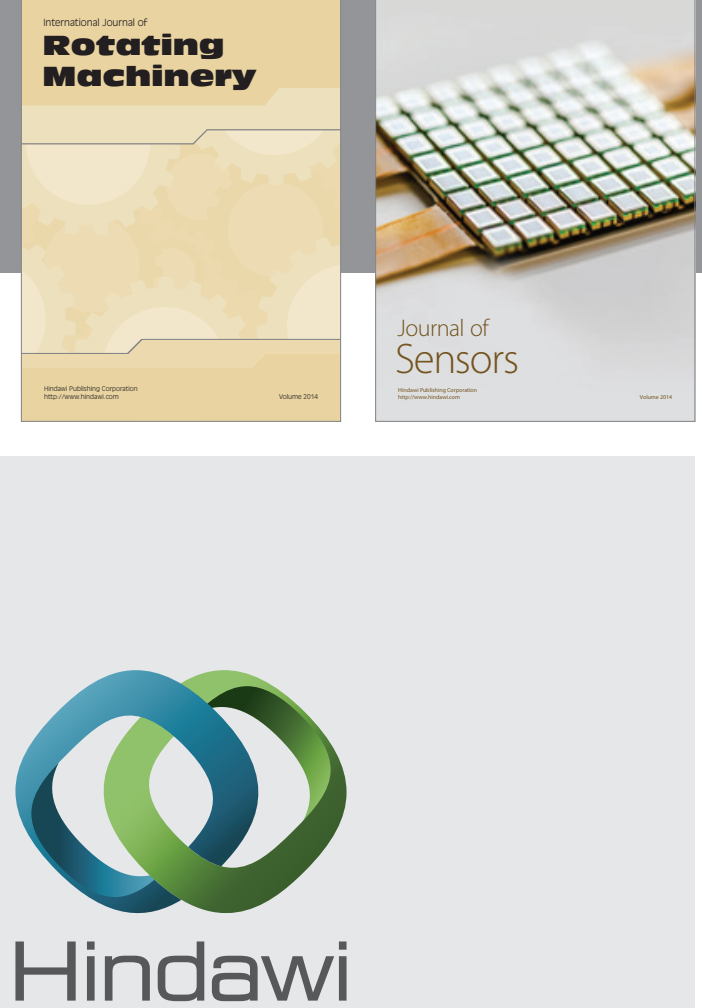

Submit your manuscripts at http://www.hindawi.com
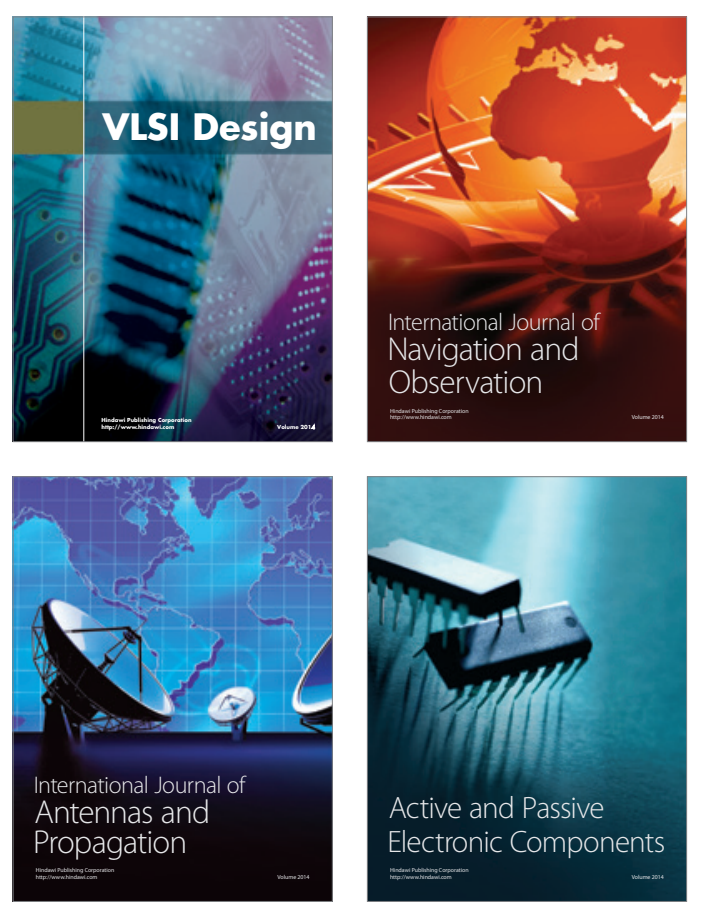
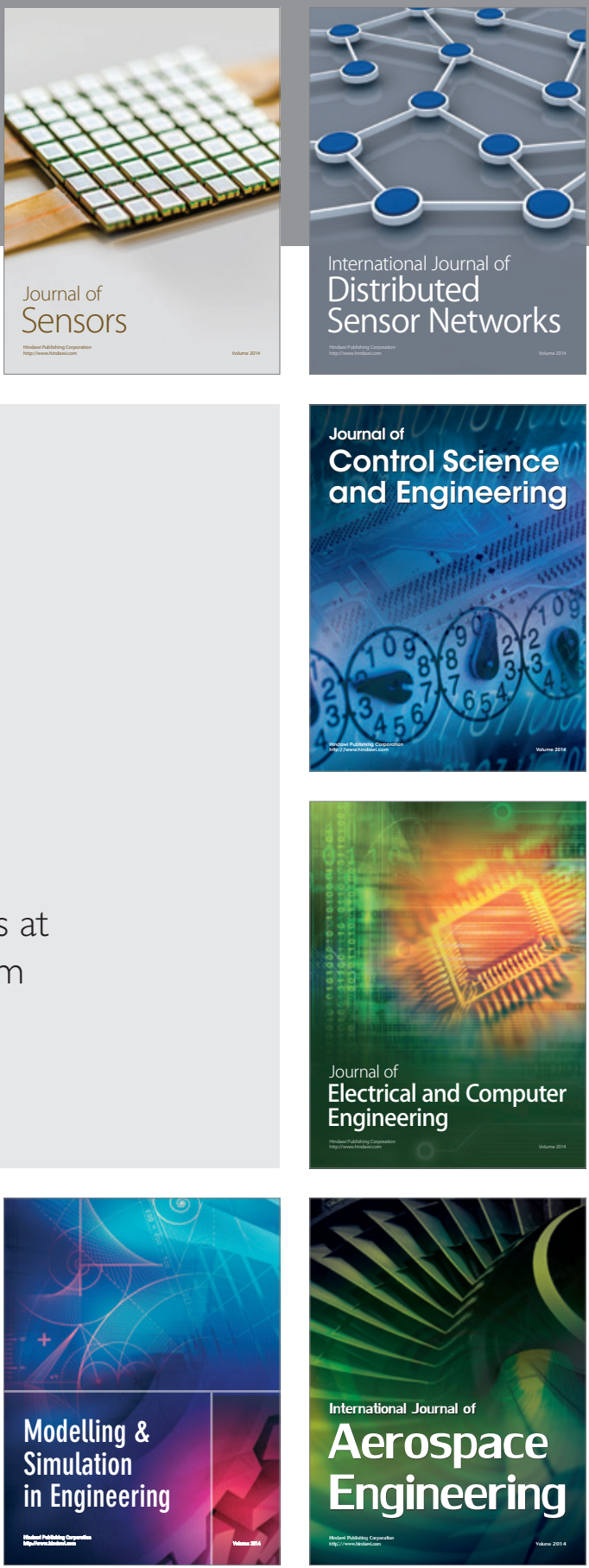

Journal of

Control Science

and Engineering
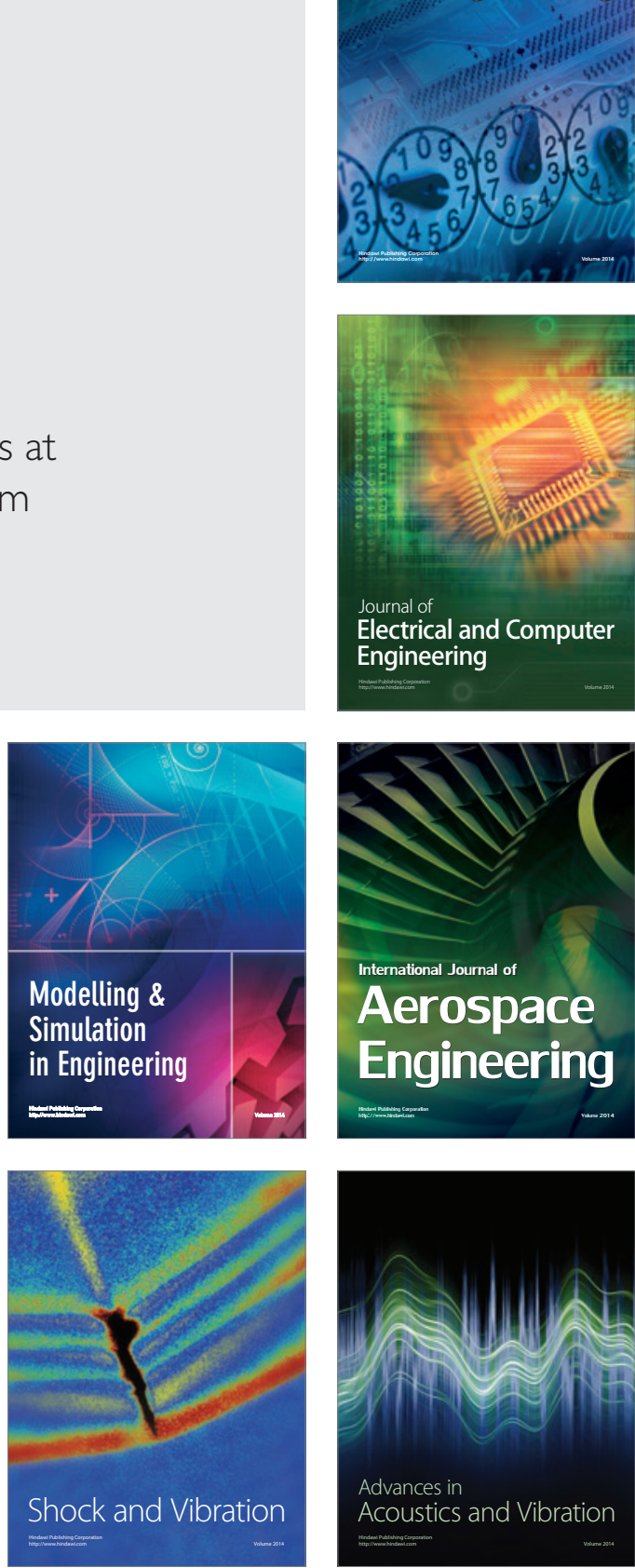